\title{
Infección vesical por esquistosoma: una causa inhabitual de hematuria
}

\author{
Borrell Palanca A, Queipo Zaragozá JA, Beltrán Meseguer JF, Chicote Pérez F, Escoms \\ Trullenque $F^{* *}$, Pastor Sempere F.
}

Servicio de Urología. *Servicio de Medicina Interna. **Servicio de Microbiología. Hospital de Valencia.

Actas Urol Esp. 2008;32(2):253-255

\section{RESUMEN}

INFECCIÓN VESICAL POR ESQUISTOSOMA: UNA CAUSA INHABITUAL DE HEMATURIA

La inmigración es un fenómeno real en nuestro país que repercute directamente en la sanidad. En la atención a estos pacientes no debemos olvidar la existencia de enfermedades inusuales en nuestro medio. Presentamos el caso clinico de un paciente con hematuria por una esquistosomiasis vesical.

Palabras clave: Schistosoma haematobium. Hematuria. Infección vesical. Inmigración.

\section{ABSTRACT}

BLADDER INFECTION BY SCHISTOSOMA: AN UNCOMMON CAUSE OF HAEMATURIA

Immigration is a real phenomenon in our country with direct consequences into the public health system. In the attendance to these patients we do not have to forget the existence unusual diseases in our setting. We presented the clinical case of a patient with hematuria caused by bladder schistosomiasis.

Keywords: Schistosoma haematobium. Haematuria. Bladder infection. Immigration.

$\mathrm{L}^{\mathrm{a}}$ a Esquistosomiasis (ó Bilharziasis) es una enfermedad parasitaria que afecta a más de 200 millones de personas en el mundo, siendo, después del paludismo, la segunda parasitosis mundial, considerada endémica en los países africanos. La infestación del adulto y la oviposición a nivel vesical determinan la aparición de una sintomatología y de unas lesiones que van a requerir un diagnóstico y tratamiento específicos. En España, los raros casos que se producen sugieren el origen importado de inmigrantes africanos y ocasionalmente en turistas que refieren una estancia previa en países de alta endemia parasitaria $^{1,2}$.

\section{CASO CLÍNICo}

Presentamos el caso de un varón de raza negra de 22 años, subsahariano, natural de Mali, que es remitido por una ONG a nuestra consulta por cuadro de hematuria macroscópica intermitente asociada a sintomas irritativos-miccionales de 3-4 años de evolución. Por medio de un traductor se llegó a la conclusión de que el paciente "veía gusanos en su orina y que nunca consultó por dicho motivo". No se pudieron conseguir más datos clínicos por las dificultades de comunicación. A la exploración física, presentaba un abdomen blando y depresible, sin defensa, ni dolor a la palpación. En el hemograma destacó una eosinofilia del $13 \%$, siendo la bioquímica y coagulación normales. En el sedimento de orina se observaron $>100$ hematies/campo. La radiografia simple de abdomen mostró una calcificación lineal de toda la pared vesical (Fig. 1) y la ecografia una lesión proliferativa intravesical sugestiva de tumor vesical (Fig. 2). Se solicitó estudio parasitológico de orina que reveló la presencia de múltiples huevos no operculados de Schistosoma haematobium con su típico espolón o espina terminal, característica de la especie (Fig. 3). Curiosamente el estudio parasitológico de heces fue negativo. 


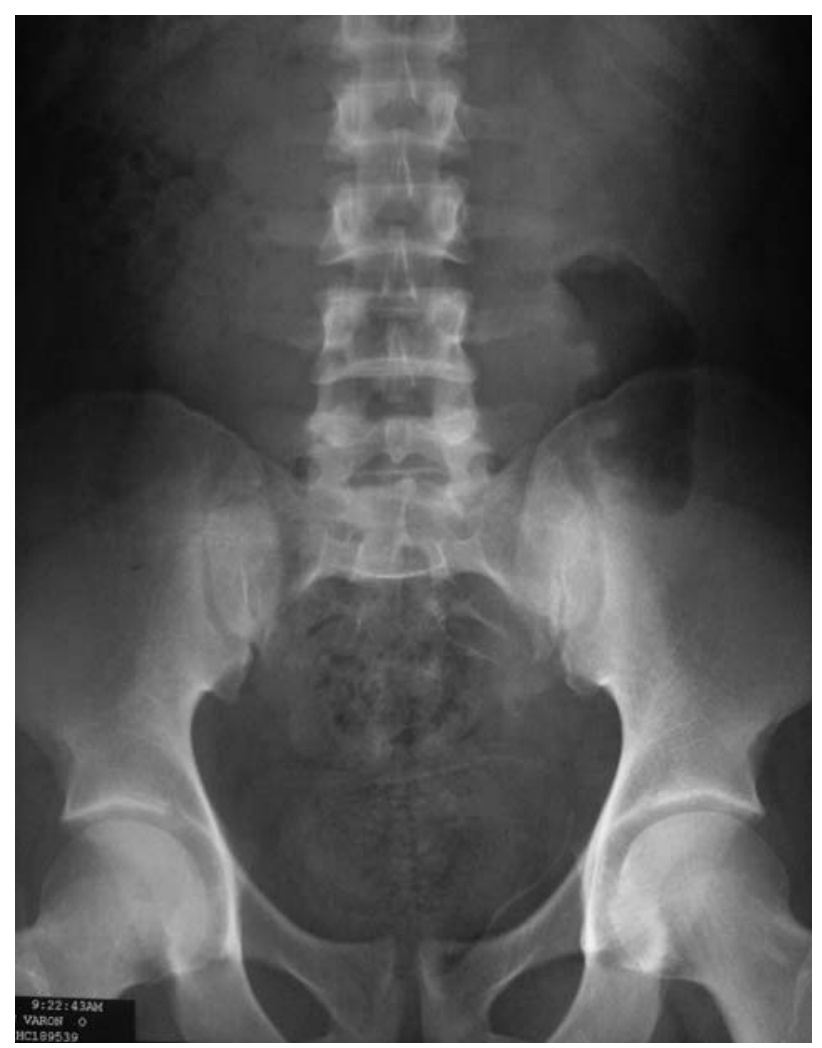

FIGURA 1. Radiografia simple de abdomen: calcificación lineal de toda la pared vesical.

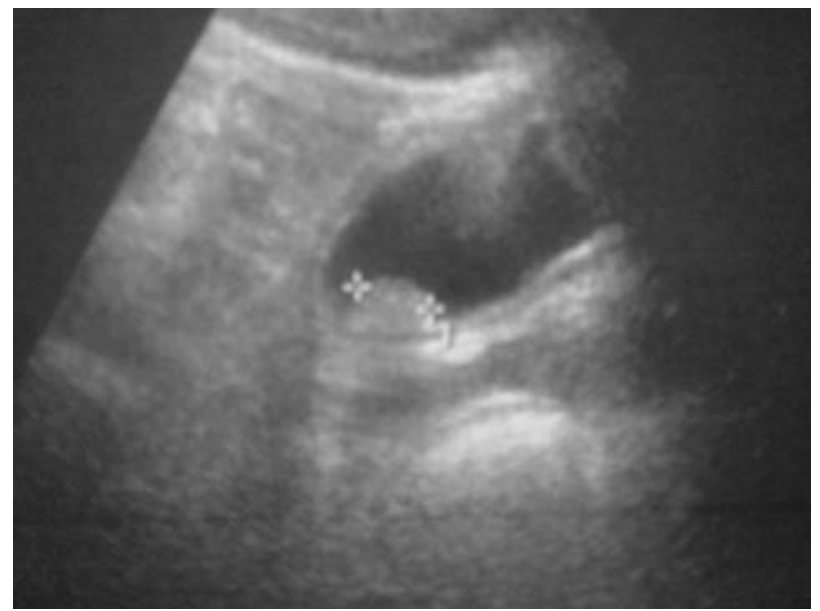

FIGURA 2. Ecografía vesical: lesión proliferativa intravesical muy sugestiva de tumor vesical.

El paciente fue tratado con Praziquantel 40 $\mathrm{mg} / \mathrm{Kg}$, administrado oral en una única dosis, que se repitió a los 15 dias por persistencia de huevos en orina. Posteriormente fue sometido a resección transuretral de múltiples lesiones nodulares intravesicales, apenas sangrantes y de extraordinaria dureza. El estudio anatomopatológico mostró, en la lámina propia, un infiltrado inflamatorio difuso

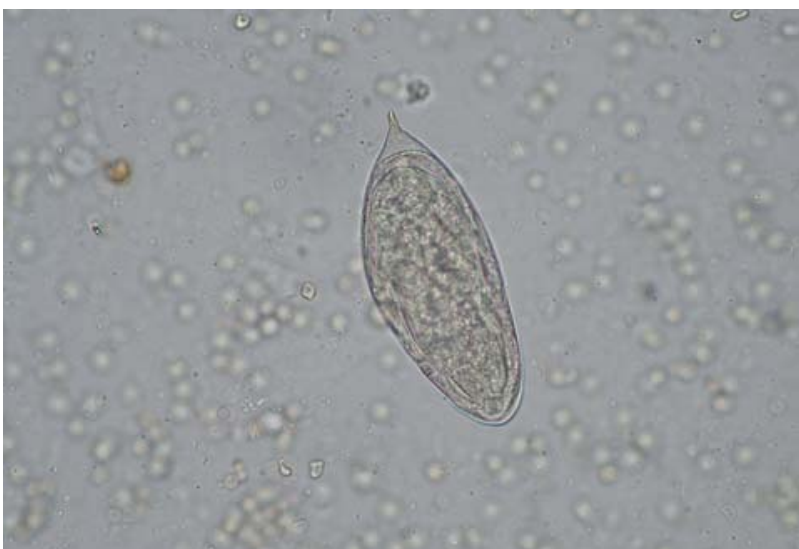

FIGURA 3. Extendido citológico de orina, en el que pueden observarse huevos de esquistosoma haematobium con su típica espina terminal. Frotis en fresco $x 400$.

con predominio de eosinófilos alrededor de huevos de Schistosoma haematobium y un infiltrado granulomatoso con focos de calcificación, alrededor de una típica sección tranversal del parásito adulto de Schistosoma haematobium "in situ" (Fig. 4).

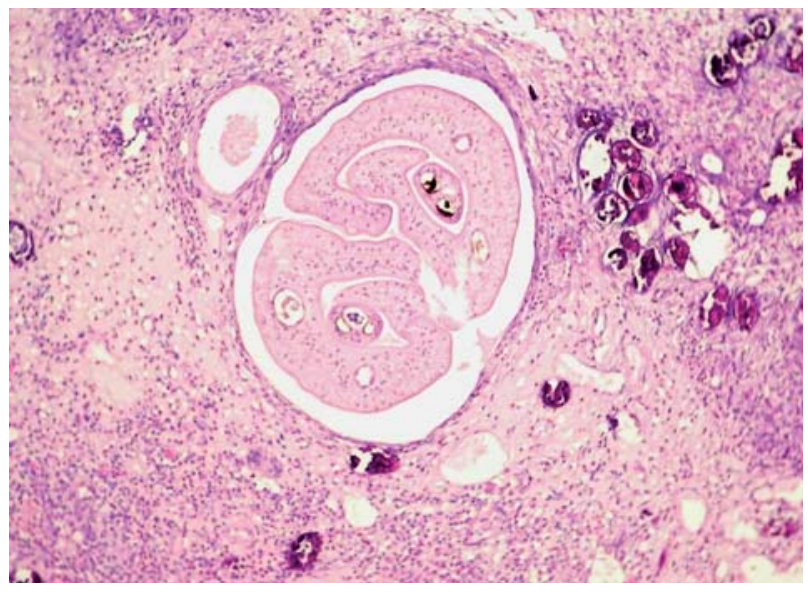

FIGURA 4. Imagen microscópica de vejiga urinaria en la que se observa un infiltrado inflamatorio difuso, focalmente granulomatoso, y focos de calcificación, alrededor de una típica sección transversal de esquistosoma haematobium "in situ". HE $x 100$.

\section{DISCUSIÓN}

La Esquistosomiasis (ó bilharziasis) es una enfermedad parasitaria producida por un trematodo platelminto del género Schistosona, que afecta tanto a varones como a mujeres, con una incidencia que alcanza su pico en edades adolescentes, entre 11 y 15 años. La infección depende del contacto de la piel con aguas dulces contaminadas como balsas y pantanos que contengan "cercarias" (formas infestantes). 
Las lesiones a nivel vesical pueden ser, además de las de carácter inflamatorio, esclerosis, calcificaciones, estenosis del cuello vesical y tardiamente cáncer vesical del tipo epidermoide ${ }^{4}$.

La hematuria, principal signo de la infestación, puede presentarse en cualquier estadio de la enfermedad. En países africanos la presencia de hematuria presenta un alto valor predictivo positivo de esquistosomiasis vesical; por ello en individuos jóvenes que provienen de zonas de alta prevalencia, la hematuria (macro o microscópica) debe hacernos pensar en esta enfermedad ${ }^{5}$. Así, Schistosoma haematobium fue el responsable del $73,7 \%$ de los casos que consultaron por hematuría en una comunidad de inmigrantes africanos de la comarca del Maresme ${ }^{6}$. La relación con la génesis del carcinoma vesical epidermoide es directa en zonas donde esta infección tiene alta prevalencia.

El tratamiento con Praziquantel a dosis de 40 $\mathrm{mg} / \mathrm{Kg}$ administrado vía oral en una única dosis o $20 \mathrm{mg} / \mathrm{Kg}$ (dos dosis en un día) es la pauta habitual. Como alternativa se puede administrar Metrifonate $5 \mathrm{mg} / \mathrm{Kg}$ vía oral en 3 dosis $^{1}$. Con ello se suele producir una regresión de la reacción inflamatoria producida por la oviposición, así como la curación de la infección en la gran mayoría de los casos. El éxito del tratamiento se determina al confirmar que los huevos desaparecen de la orina. Las lesiones proliferativas intravesicales deben ser resecadas para información histológica y reducir los sintomas irritativos miccionales así como la hematuria.

Uno de los propósitos de este trabajo ha sido el de concienciar a los urólogos de nuestro medio de la necesidad de tener presentes enfermedades tropicales que hasta ahora eran muy infrecuentes pero que debido al aumento de los viajes intercontinentales y el asentamiento creciente en algunas áreas de nuestro país de inmigrantes que proceden de zonas endémicas, favorecen la posibilidad de que nos encontremos, en la práctica diaria, ante pacientes que pueden tener una enfermedad parasitaria importada. En particular la esquistosomiasis es una de las enfermedades tropicales prioritarias para la Organización Mundial de la Salud. Así, se considera necesario realizar análisis de orina con estudio parasitológico del sedimento urinario a aquellos inmigrantes procedentes del África subsahariana que presentan cualquier sintoma urinario, para detectar numerosos casos de bilharziasis vesical que de lo contrario podrian pasar inadvertidos.
La mayoria de casos ocurridos en España son importados; los casos "agudos" por turistas de países con alta endemia parasitaria y los casos "crónicos" por los colectivos de inmigrantes africanos; favorecida además la parasitación en este último colectivo por las condiciones de vida de los inmigrantes en nuestro país (situación legal irregular, falta de asistencia sanitaria, desconocimiento del idioma, etc.) que dificultan el diagnóstico y tratamiento en los portadores del trematodo.

Como conclusión y dada la elevada prevalencia de la esquistosomiasis en los países subsaharianos y sus posteriores implicaciones clínicoepidemiológicas, la aparición de una hematuria macro o microscópica en inmigrantes de raza negra, o incluso la aparición de cualquier sintomatología urológica, necesita una evaluación completa, al menos con un estudio parasitológico que considere la bilharziasis como la causa más probable. Y no olvidar que los viajes de aventura de los turistas españoles con destinos exóticos, obliga a pensar en la esquistosomiasis y otras parasitosis tropicales como enfermedades que cada vez tendrán mayor relevancia en nuestra práctica médica.

\section{REFERENCIAS}

1. Bichler KH, Savatovsky I; the Members of the Urinary Tract Infection (UTI) Working Group of the Guidelines Office of the European Association of Urology (EAU): Naber KG, Bischop MC, Bjerklund-Johansen TE, Botto H, et al. Guidelines for the Management of Urogenital Schistosomiasis. Eur Urol. 2006;49(6):998-1003.

2. Cocharan M, Almeda J, Vinuesa T, Valis ME, Mejías T, Jou $\mathrm{P}$, et al. Esquistosomiasis importada por viajeros españoles: estudio clínico epidemiológico de 80 casos. Med Clin. 1997; 108(7):721-725.

3. Ross AG, Bartley PB, Sleig AC, Olds GR, Li Y, Williams GM, et al. Schistosomiasis. N Engl J Med. 2002;346(16):12121220.

4. Vázquez Villegas J, Galindo Pelayo JP, Gámez Gámez E. Asistencia inicial a inmigrante en atención primaria. FMC. 2003;10(5):83-97.

5. Amole Bo, Jinadu MK. Urinary schistosomiasis among school children in Ile-Ife, Nigeria. Afr J Med Med Sci. 1994; 23(3):249-252.

6. Roca C, Balanzó X, Fernández JL, Pujol E, Corachan M. Caracterización demográfica, motivos de consulta y morbilidad prevalente en una comunidad de inmigrantes africanos de la comarca del Maresme. Med Clin. 1999;112(7):215-217.

Correspondencia autor: Dr. A. Borrell Palanca

Servicio de Urología. Hospital de Sagunto

Ramón y Cajal, s/n - 46520 Sagunto (Valencia)

E-mail autor: borrell_alb@gva.es

Información artículo: Nota clínica

Trabajo recibido: julio 2006

Trabajo aceptado: septiembre 20067 